\title{
Influences of geometry of particles on electrorheological fluids
}

\author{
Yabing Qi and Weijia Wen \\ Department of Physics, The Hong Kong University of Science and Technology, Clear Water \\ Bay, Kowloon, Hong Kong, People's Republic of China
}

Received 13 June 2002

Published 16 August 2002

Online at stacks.iop.org/JPhysD/35/2231

\begin{abstract}
Electrorheological (ER) fluids with microspheres and micro-rods as the component solid phase were examined experimentally to investigate the influences of particle geometry on ER performance. It is observed that for both dried and water-activated ER fluids, the shape of the constituent particles plays a significant role, i.e. for particles with same diameters, the microsphere-based ER fluids show better ER performance than micro-rod-based ER fluids. We found from experiments that the aspect ratio of particles is an important parameter for the ER activities. The tendency is that the ER effect decreases with the increase of the aspect ratio, while this phenomenon becomes much weaker in the case when dried particles were substituted for the ones with moisture.
\end{abstract}

\section{Introduction}

Electrorheological (ER) fluids consist of dielectric solid particles suspended in a relatively non-conductive liquid [1-4]. Upon the application of an electric field, the apparent viscosity of the ER fluid can be changed by several orders of magnitude, due to the formation of chain-like or column-like structures along the direction of the external electric field. The response time is of the order of milliseconds, and the process is reversible, i.e. when the electric field is removed, the structures vanish and the colloid returns to its original state. The ability to electrically control the viscosity renders ER fluids potentially useful in numerous electro-mechanical devices [5-7].

Although ER fluids have been known for decades [8], and many new ER fluids have been developed, the physical mechanism of the ER effect has been under investigation in the past years [9-13]. Among various models proposed to interpret the behaviour of ER fluids, an important one is the dipole approximation, which gives the quadratic relation between the interaction force $f_{\mathrm{d}}$ and polarizability $\beta$, i.e. $[14,15]$

$$
f_{\mathrm{d}} \propto R^{6}|\beta|^{2}
$$

where $\mathrm{R}$ is the radius of solid particles, and

$$
\beta=\frac{\tilde{\varepsilon}_{\mathrm{p}}-\tilde{\varepsilon}_{\mathrm{f}}}{\tilde{\varepsilon}_{\mathrm{p}}+2 \tilde{\varepsilon}_{\mathrm{f}}}
$$

where $\tilde{\varepsilon}_{\mathrm{p}}=\varepsilon_{\mathrm{p}}-\mathrm{i}\left(\sigma_{\mathrm{p}} / \omega \varepsilon_{0}\right)$ and $\tilde{\varepsilon}_{\mathrm{f}}=\varepsilon_{\mathrm{f}}-\mathrm{i}\left(\sigma_{\mathrm{f}} / \omega \varepsilon_{0}\right)$.
It is obvious that the ER effect is determined by many factors, such as the dielectric properties of the two phases, the electric field strength as well as the volume fraction. Except for the factors mentioned above, it was found that the particle geometry might play a significant role in ER effect $[16,17]$. The influence of particle shape on the ER effect was also carried out recently and the results indicated that maximum of yield stress was strongly depending on the particle shape [18]. In this paper, we present some experimental results with regard to the influence of the particle shape on ER performance under $\mathrm{DC}$ and $\mathrm{AC}$ electric fields.

\section{Experiments}

Glass microspheres (SIGMA CHEMICAL CO.) and glass fibres (CUDA PRODUCTS CORP.) were used as dispersed particles in the silicone oil (density $\rho=900 \mathrm{~kg} \mathrm{~m}^{-3}$ ). Glass microspheres were selected by sieves, and separated with respect to their diameters. Glass fibres were cut into desired lengths with a diamond cutter.

Two series of ER fluids were prepared including dried and water-activated samples. Before the preparation of samples, both particles and oil were heated at $120^{\circ} \mathrm{C}$ for about $3 \mathrm{~h}$ to remove any trace water. The dried microspheres with different diameters $(D=25$ and $50 \mu \mathrm{m})$ and micro-rods with different aspect ratios (diameter $D=50$ and $75 \mu \mathrm{m}$, length $L=260$ and $390 \mu \mathrm{m}$ ) were mixed with silicone oil, respectively. In order to compare the ER effect at fixed volume fraction all 
samples are prepared with volume fraction 0.2 ; however, other ER fluid samples with lower volume fractions are also tested. To prepare water-activated ER fluids, the dried particles were immediately put into a glass bottle, into which an amount of water $(0.5 \%$ by weight) was added with a syringe (HAMILTON CORP.). The weights of respective dried particles and water were measured by an electronic balance (OHAUS CORP. ARRV70). The bottle was then sealed and kept at $120^{\circ} \mathrm{C}$ for $5 \mathrm{~h}$. After cooling down to room temperature, a measured amount of silicone oil was injected into the bottle. Then the ER fluids were stirred to ensure that the water-coated particles mixed with the oil thoroughly.

With a cell comprising two parallel electrodes, the dielectric measurements were carried out with a HP4192A LF impedance analyser. The rheological properties were measured by a plate/plate viscometer (Haake RS1) with gap width $1 \mathrm{~mm}$, and experimental data were collected with software Rheowin. The power supply was specially designed to provide DC and sinusoidal AC electric voltage outputs. All experiments were done at room temperature.

\section{Results and discussion}

The microscopic pictures for the alignment of particles in ER fluids, carried out with a volume fraction of about 0.072 , are shown in figures $1(a)$ and $(b)$ captured by an optical microscope with a charge-coupled-device camera. Seen from figure 1(a), the microspheres align in the direction of the external electric field to form chain-like structures between two electrodes, while figure $1(b)$ shows the alignment for micro-rods in ER fluids. We observed that when an electric field is applied, each micro-rod turned its long axis parallel to the direction of the external field and interacted with its neighbouring micro-rods to form chain-like structures. However, we also noted that the chains formed by micro-rods were not perfectly aligned and some cross-links among the rods could be seen.

(a)

(b)
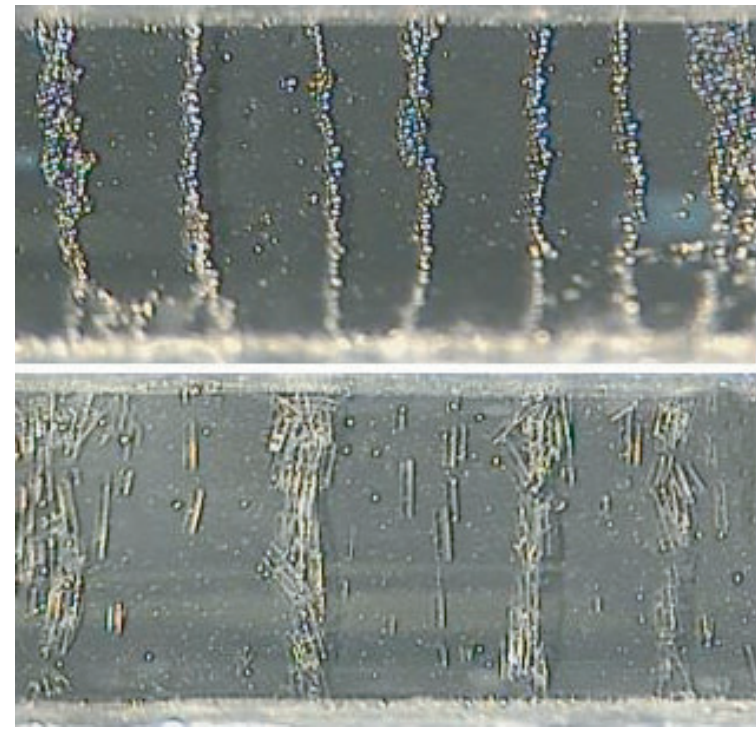

Figure 1. The chain-like structures in the host silicone oil formed by $(a)$ microspheres and $(b)$ micro-rods in the presence of an external electric field.
The dielectric properties of dried glass particles can be found in [14]. The results of measurements of the dielectric property for the water-activated ER fluids are plotted in figures 2 and 3 . It can be seen from figure 2 that the dielectric constants of the water-activated ER fluids decrease with increase in frequency, while the dielectric constant of the silicone oil remains almost constant at 3.2 over the whole tested frequency range from $200 \mathrm{~Hz}$ to $100 \mathrm{kHz}$. From figure 3, it can be seen that for all water-activated ER fluids and silicone oil, the conductivity increases with increase in frequency. We also note from figures 2 and 3 that both dielectric constants and conductivities measured with microsphere-based ER fluids are much larger than those carried out with ER fluids composed of micro-rods. In addition, for the micro-rod-based ER fluids the dielectric properties are strongly affected by the aspect ratio of the particle. The relations of the dielectric properties of wateractivated ER fluids versus aspect ratio $L / D$ at fixed frequency $10 \mathrm{kHz}$ are shown in the insets of figures 2 and 3, which show that both the dielectric constant and the conductivity decreases with increase in aspect ratio. This phenomenon might result from the differences of the particle surface area on which the water is coated.

Figure 4 shows the shear stresses of water-activated ER fluids as a function of the DC electric field strength at a constant shear rate $\dot{\gamma}=5 \mathrm{~s}^{-1}$. For all the water-activated ER fluids, the shear stress increases almost linearly with the increase of the DC electric field strength. The plot of the shear stresses versus aspect ratio at fixed field strength $2200 \mathrm{~V} \mathrm{~mm}^{-1}$ is shown in the inset of figure 4, from which we can see that the shear stress decreases with increase in aspect ratio. The origin of the

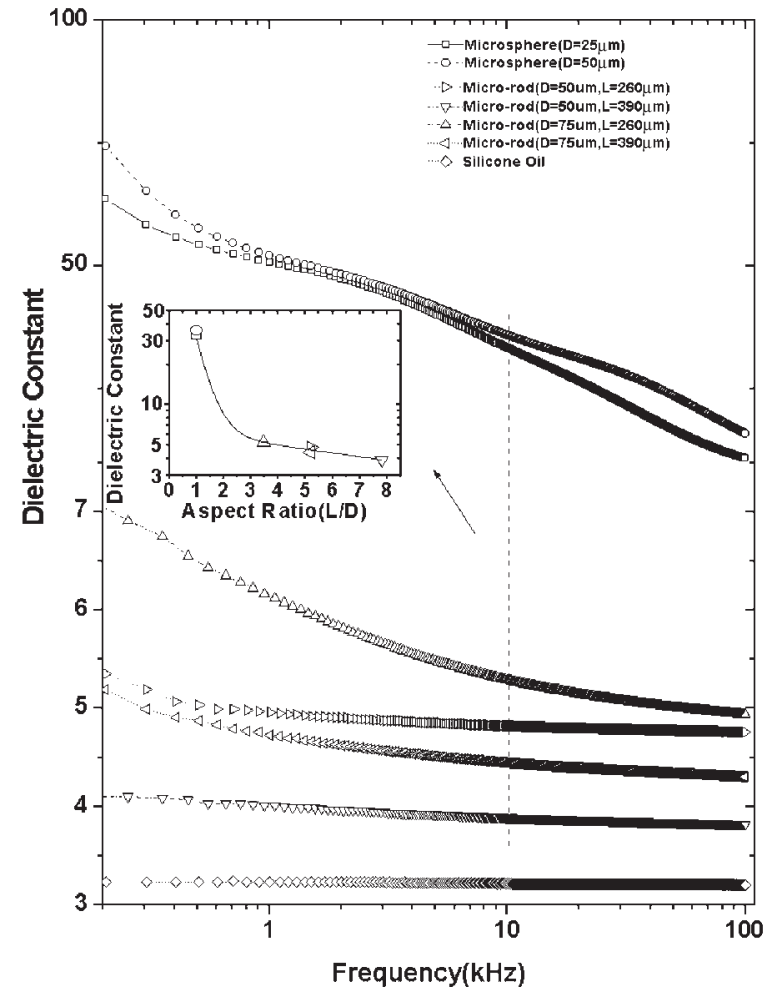

Figure 2. Dielectric constants of the water-activated ER fluids and silicone oil as a function of the frequency. Inset gives dielectric constant versus aspect ratio $(L / D)$ at fixed frequency $f=10 \mathrm{kHz}$, and the line across the symbols is a guide to the eyes. 


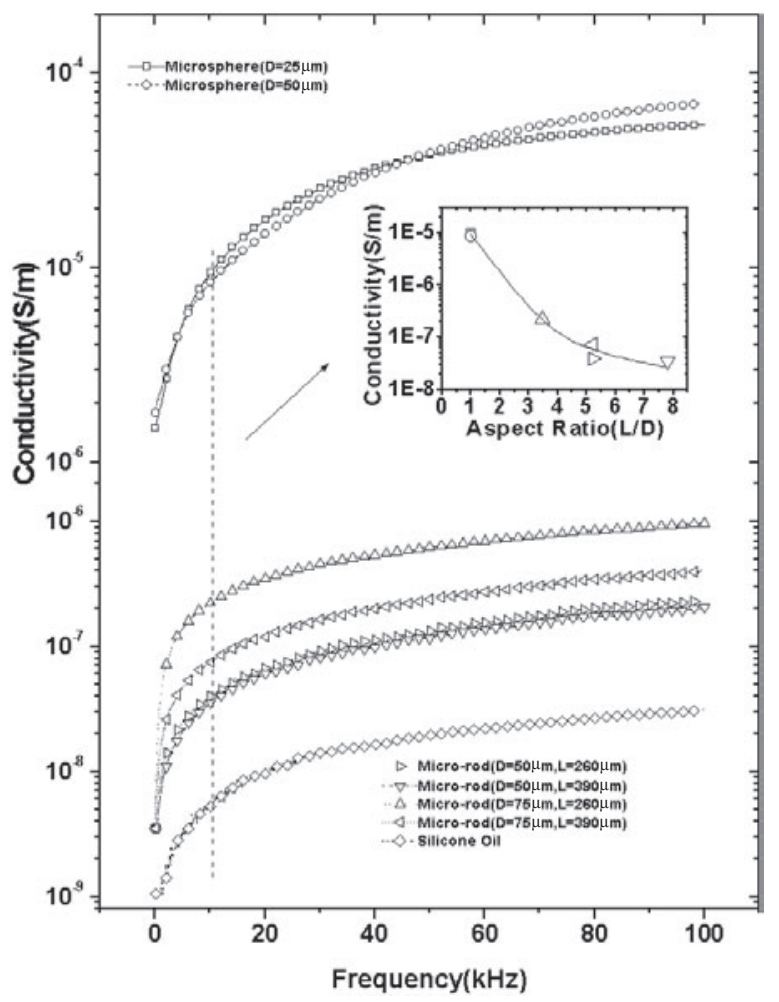

Figure 3. Conductivities of the water-activated ER fluids and silicone oil as a function of the frequency. Inset gives conductivity versus aspect ratio $(L / D)$ at fixed frequency $f=10 \mathrm{kHz}$, and the line across the symbols is the guide to the eyes.

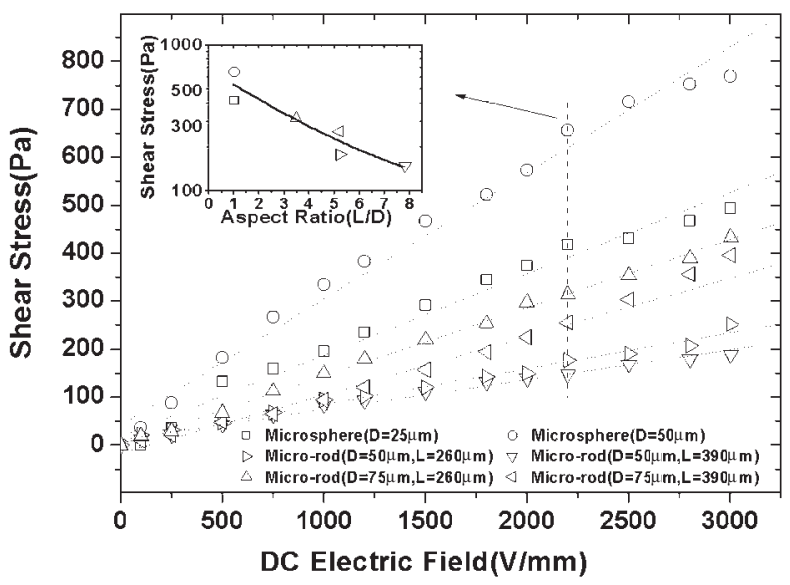

Figure 4. Shear stresses of the water-activated ER fluids versus DC electric field strength at fixed shear rate $\dot{\gamma}=5 \mathrm{~s}^{-1}$. The dotted lines are the linear fittings for the experimental data. The inset gives the dependence of shear stress on aspect ratio $(L / D)$ at fixed field strength $2200 \mathrm{~V} \mathrm{~mm}^{-1}$, and the line across the symbols is a guide to the eyes.

tendency might be ascribed to the surface effect as mentioned above, i.e. for particles with the same volume, the particles with smaller aspect ratios will have more surface area than those with larger aspect ratios, and thus have more surface moisture. The inset of figure 4 is similar to that for the insets of figures 2 and 3, which indicates that the ER effect is closely related to the dielectric properties of ER fluids $[13,19]$. One thing that should be emphasized here is that although the aspect ratios for

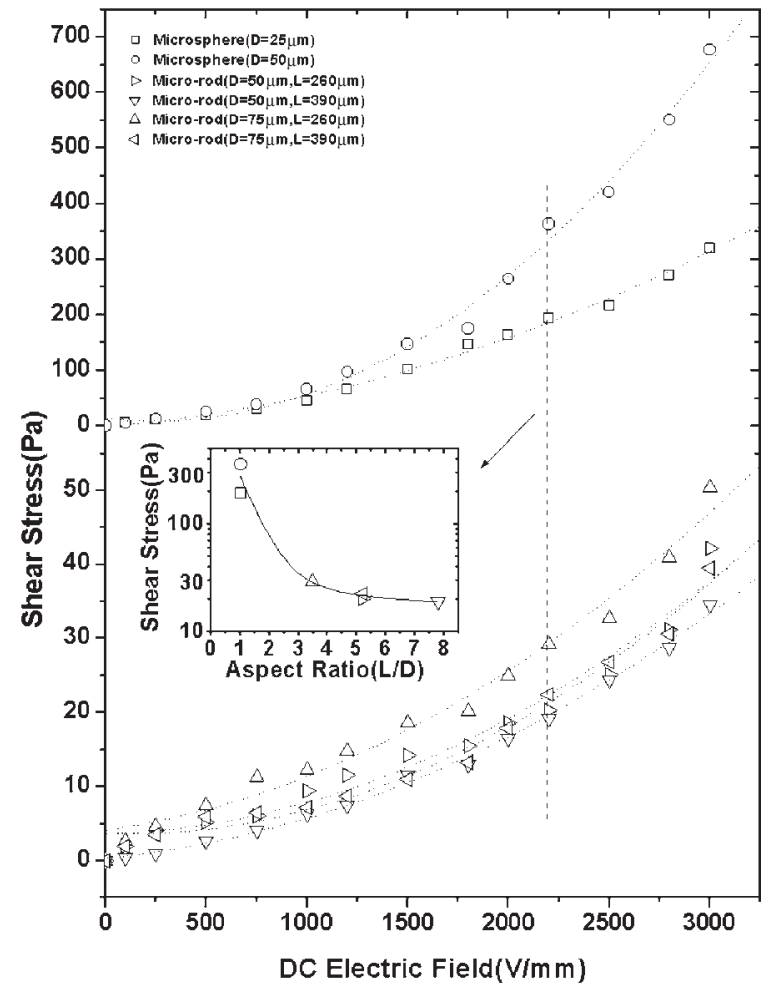

Figure 5. Shear stresses of the dried ER fluids versus DC electric field strength at fixed shear rate $\dot{\gamma}=5 \mathrm{~s}^{-1}$. The dotted lines are the second order polynomial fittings for the experimental data. The inset gives the dependence of shear stress on aspect ratio $(L / D)$ at fixed field strength $2200 \mathrm{~V} / \mathrm{mm}$, and the solid line across the symbols is the guide to the eyes.

the two microsphere samples $(D=25$ and $50 \mu \mathrm{m})$ and the two micro-rod samples $(D=50 \mu \mathrm{m}, L=260 \mu \mathrm{m} ; D=75 \mu \mathrm{m}$, $L=390 \mu \mathrm{m}$ ) are the same, the larger the particles that are used the stronger the ER effect that can be obtained.

In order to investigate the influence of water on the ER performance, the dried particles were substituted for ones with moisture and the results are shown in figure 5, where the shear stresses for the dried ER fluids are plotted as a function of DC electric field strength. We found from the experiments that the major difference between figures 5 and 4 is that for dried ER fluids; the shear stress shows a nearly quadratic relation with field strength, while the relation is almost linear for their water-activated counterparts. In the inset of figure 5 the shear stress for the dried ER fluids is plotted against the aspect ratio at fixed field strength $2200 \mathrm{~V} \mathrm{~mm}^{-1}$. By comparing the insets of figures 5 and 4, it can be seen clearly that without the wateractivation process, the shear stress becomes smaller, especially for the micro-rod-based ER fluids. The physical mechanism for this is not completely understood to date; however, it can be seen from our experiments that both the dielectric constant and conductivity of watered ER fluids increased by a larger amount when compared with those of water-free ER fluids, which may somehow be intrinsically connected to the ER performance. An interesting feature is identified from the insets of figures 4 and 5, namely that the shear stresses for the microspherebased ER fluids are much larger than those for the microrod-based ER fluids. One possible reason is that in dynamic measurements, it is easy for the micro-rods to tangle together 


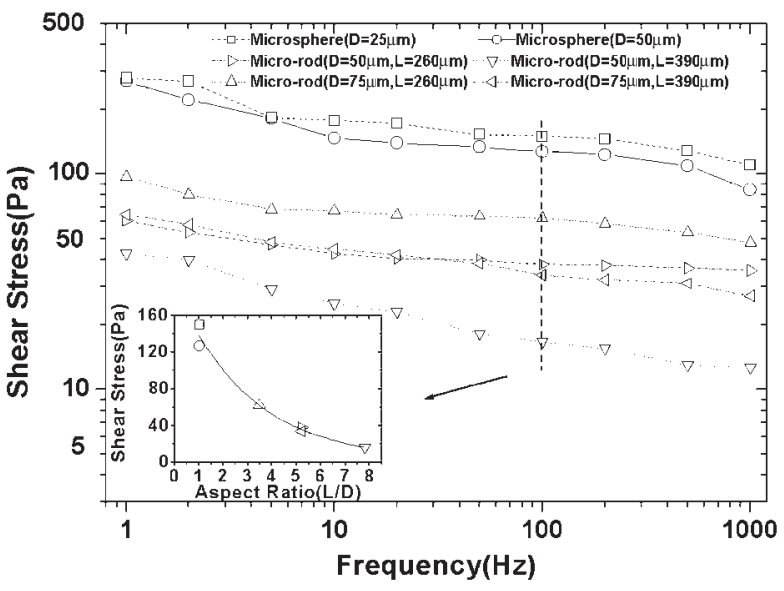

Figure 6. Shear stresses of the water-activated ER fluids versus AC electric field frequency, when shear rate $\dot{\gamma}=5 \mathrm{~s}^{-1}$ and AC electric field strength (peak-peak value) $\mathrm{ACpp}=2000 \mathrm{~V} \mathrm{~mm}^{-1}$. The inset gives the dependence of shear stress on aspect ratio $(L / D)$ at fixed frequency of $100 \mathrm{~Hz}$, and the line across the symbols is a guide to the eyes.

between the two parallel plates, and thus it is difficult for the micro-rods to align well in the direction of the external electric field, as can be seen from figure $1(b)$, especially for the case of low field situation. It is obvious that one cannot expect a large ER effect if chain structures are not perfectly constructed. Except for the particle size and shape, our experimental results also indicate that the ER effect strongly depends on the volume fraction; for example, the dried rods show every weak ER performance when the volume fraction decreases below 0.1 . Additional samples with similar rods (diameter $=50 \mu \mathrm{m}$, length $=260 \mu \mathrm{m}$ ) but different volume fractions of $0.15,0.1$ and 0.075 , respectively, were employed for comparison, and corresponding shear stresses measured at a field of $2 \mathrm{kV} \mathrm{mm}^{-1}$ were 10.4, 8.6 and 5.7 Pa. It should be pointed out that the fluctuation in readings became serious when the testing was carried out with low volume fraction.

Figure 6 shows the shear stresses for the water-activated ER fluids versus frequency of the electric field under fixed strength of $2000 \mathrm{~V} \mathrm{~mm}^{-1}$, where the shear stress decreases for all samples as the frequency increases. A similar phenomenon can be seen if the water-activated particles are replaced with dried ones, which is shown in figure 7. However, we can observe from figurs 6 and 7 that the variations in the shear stresses for the dried micro-rod ER fluids are too small to be identified compared to those for water-activated particles. The influences of the aspect ratios on the shear stress for both wateractivated and dried particles are shown in the insets of figures 6 and 7 , respectively, which indicate that the larger the aspect ratio the smaller the ER effect observed.

Figure 8 shows that the shear stresses for the dried ER fluids increases as the AC electric field strength increases, at fixed frequency $10 \mathrm{~Hz}$ and constant shear rate, where the dependence of shear stress on the aspect ratio at fixed field strength is shown as an inset. It should be pointed out that an interesting common feature captured from the insets in figures $6-8$ is that a stronger ER effect can be obtained if smaller microspheres are used under an AC field, which is totally different from that carried out under a DC field (see figure 5 for details).

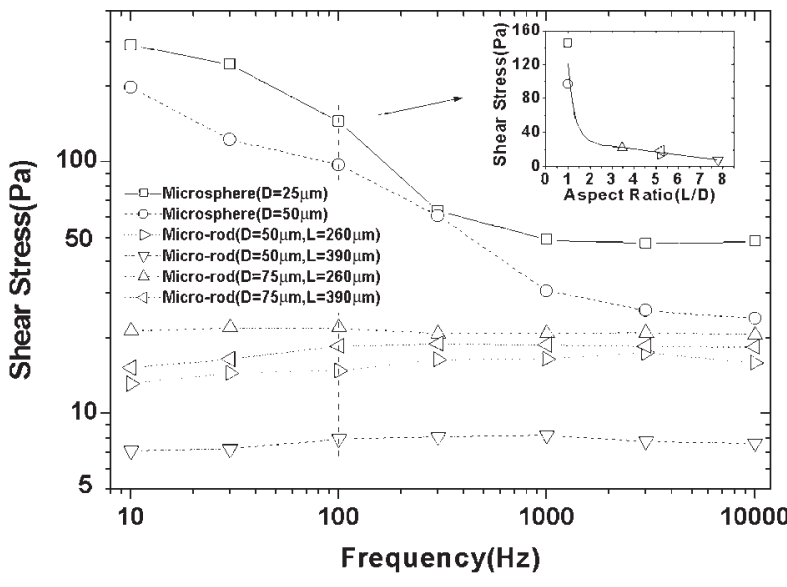

Figure 7. Shear stresses of the dried ER fluids versus AC electric field frequency, when shear rate $\dot{\gamma}=5 \mathrm{~s}^{-1}$ and AC electric field strength (peak-peak value) ACpp $=3000 \mathrm{~V} \mathrm{~mm}^{-1}$. The inset gives the dependence of shear stress on aspect ratio $(D / L)$ at fixed frequency of $100 \mathrm{~Hz}$, and the line across the symbols is a guide to the eyes.

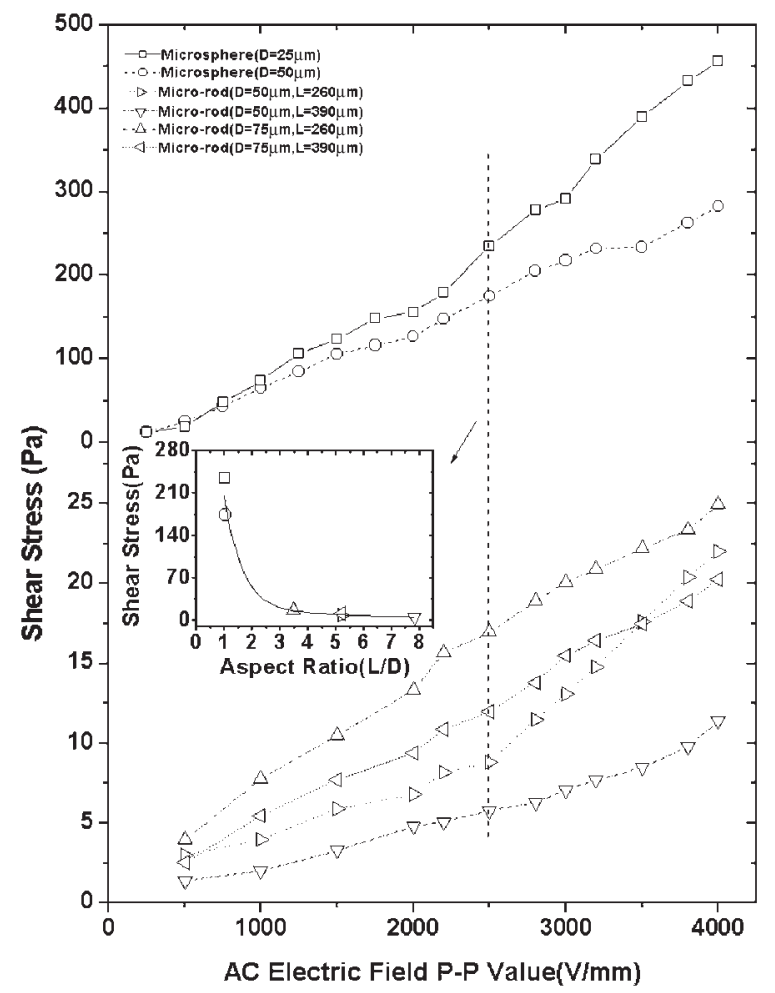

Figure 8. Shear stresses of the dried ER fluids versus AC electric field strength at fixed shear rate $\dot{\gamma}=5 \mathrm{~s}^{-1}$, when AC electric field frequency is constant at $10 \mathrm{~Hz}$. The inset gives the dependence of shear stress on aspect ratio at fixed AC electric field strength $2500 \mathrm{~V} \mathrm{~mm}^{-1}$, and the line across the symbols is a guide to the eyes.

\section{Conclusion}

In this paper, we prepared ER fluids with microspheres and micro-rods as the component solid phase. The dielectric properties, DC and AC ER effects were examined to investigate the influence of particle geometry on ER fluids. It is observed that for both the dried and water-activated ER fluids, the shape 
of the constituent particles plays a significant role, and the microsphere-based ER fluids show better ER performance than the micro-rod-based ER fluids; for the micro-rod-based ER fluids, the aspect ratio is an important factor for water-activated samples, i.e. ER effect of micro-rod ER fluids decreases with increase in aspect ratio, and the influence becomes much weaker in the case of dried samples.

\section{Acknowledgment}

We acknowledge support from CERG HKUST6152/00P, the National Natural Foundation of China and the William Mong Solid State Cluster Laboratory.

\section{References}

[1] Halsey T C and Toor W 1990 Phys. Rev. Lett. 652820

[2] Tao R and Sun J M 1991 Phys. Rev. Lett. 67398

[3] Ma H, Wen W, Tam W Y and Sheng P 1996 Phys. Rev. Lett. 772490

[4] Wen W, Ma H, Tam W Y and Sheng P 1998 Appl. Phys. Lett. 733070

[5] Block H and Kelly J P 1988 J. Phys. D: Appl. Phys. 211661
[6] Sims N D, Stanway R, Peel D J, Bullough W A and Johnson A R 1999 Smart Mater. Struct. 8601

[7] Hao T 2001 Adv. Mater. 131847

[8] Winslow W M 1949 J. Appl. Phys. 201137

[9] Davis L C 1992 Appl. Phys. Lett. 60319

[10] Wen W, Ma H, Tam W Y and Sheng P 1997 Phys. Rev. E 551294

[11] Tam W Y, Yi G H, Wen W, Ma H, Loy M M T and Sheng P 1997 Phys. Rev. Lett. 782987

[12] Wu C W and Conrad H 1997 J. Phys. D: Appl. Phys. 302634 Wu C W and Conrad H 1998 J. Appl. Phys. 813880

[13] Ikazaki F, Kawai A, Uchida K, Kawakami T, Edamura K, Sakurai K, Anzai H and Asako Y 1998 J. Phys. D: Appl. Phys. 31336

[14] Wen W and Lu K 1996 Appl. Phys. Lett. 683659

[15] Lan Y C, Men S Q, Xu X Y and Lu K Q 2000 J. Phys. D: Appl. Phys. 331239

[16] Gast A P and Zukoski C F 1989 Adv. Colloid Interface Sci. 30153

[17] See H and Tamura H and Doi M 1993 J. Phys. D: Appl. Phys. 26746

[18] Duan X, Luo W, Chen H and He Y 2000 J. Intel. Mat. Syst. Str. 1143

[19] Lan Y, Xu X, Men S and Lu K 1998 Appl. Phys. Lett. 732908 